\title{
Sequestration of carbon dioxide into building products based on the dolomite binder of carbonate hardening
}

\author{
Tamara Bakhtina*, Nikolay Lyubomirskiy, and Aleksandr Bakhtin \\ V.I. Vernadsky Crimean Federal University, Kievskaya Street, 181, Simferopol, 295050, Russia
}

\begin{abstract}
The paper covers the method of obtaining efficient structural heat-insulating and heat-insulating construction products based on sawdust and lime binder hardening in the medium of carbon dioxide. Building composites based on wood-cement compositions have several advantages compared to traditional concrete products, but at the same time there are several features such as incompatibility of cement binder with a certain type of wood due to the release of extractive substances by the wood and retardation of the binder which complicate the receiving technology. The use of a binder that was not affected by extractives, such as dolomite carbonate binder, greatly simplifies the production of finished products. Performed researches have shown the possibility of obtaining light building materials based on sawdust and lime binder of carbonate hardening. The process of obtaining samples in a specially designed mold takes 4-8 minutes; after that the samples achieve the required characteristics. The samples extracted from the mold do not require additional exposure and have specified geometrical and physicomechanical parameters.
\end{abstract}

\section{Introduction}

Resource and energy saving is one of the priority directions of development of science and technology. This direction can be fully realized in the building materials industry due to the production of less energy-intensive binders and the manufacture of effective structural heatinsulating and heat-insulating construction products on their basis with the involvement of various secondary raw materials in the production cycle. Such raw materials, in particular, include dolomite rocks which are one of the most common and underdeveloped varieties of mineral raw materials, as well as waste of the wood processing industry in the form of wood chips.

Today energy efficiency of buildings is one of the main indicators in the construction of buildings and structures [1]. To improve the energy efficiency of buildings, both passive and active methods for regulating the state of the internal environment are used [2]. Active methods involve the optimization of the functioning of heating and hot water systems, and passive methods are aimed at energy saving through the use of efficient structural heatinsulating and insulating building materials and products. Passive methods aimed at

\footnotetext{
* Corresponding author: t.bakhtina83@gmail.com
} 
reducing heat loss in a building are taken as a basis at the initial stage of building design [3].

Wood has been one of the most common types of materials used in construction since ancient times. Due to its physico-mechanical properties and widespread availability, a wide range of building materials and structures is produced from tree species. Sawing waste and waste of wood processing - sawdust, shavings and fibers are also a valuable base for the production of materials for construction. The list of such products is quite wide, but the technology is based on the principle of gluing wood waste into slabs, panels or blocks using various binders [4-6]. Organic and inorganic adhesives are used as such binders. Synthetic organic adhesives contain volatile compounds and are toxic, and their recycling is quite complicated [7]. Biopositive organic adhesives are more difficult to use and have a high cost [8]. From inorganic binders, portland cement, gypsum, lime and magnesia binders are used for the production of wall materials on organic fillers. Production of wall blocks based on portland cement with organic filler began in the early 20th century. They were intended for low-rise and agricultural construction. Starting in the 70s of the 20th century production of wood-cement panels began. Of course, panels based on organic binders are made in much larger quantities, but wood-cement panels have a low cost and are considered an important contribution to alleviating the housing problem in developing regions. The experience of using such materials has shown that wood-cement composites have a number of advantages over concrete elements, such as low density and excellent performance in terms of resistance to moisture, fire, mold and insects compared to ordinary wooden elements. The first developed technologies for the production of wood-cement composites differed in the duration of curing of products during hardening which could reach 24 hours. Later, methods were developed to accelerate the hardening method by pumping $\mathrm{CO}_{2}$ into a hardening formation in a gaseous or liquid form which made it possible to carry out the removal of products from the press in a few minutes [9]. Also in the production of woodcement composites, there are a number of difficulties associated with slowing down the hydration of cement due to the extraction of various water-soluble substances (mainly sugars) by the wood. The effect of extractive substances can lead not only to a decrease in strength, but also to a violation of the physical integrity of the samples. Therefore, to prevent incompatibility of wood with cement, it is necessary to carry out preliminary preparation of wood filler which consists either in soaking it or in processing it with various substances to create an alkaline environment. The presence of this operation significantly complicates and slows down the production process, so the use of other binders that are not subject to the influence of extractive substances can simplify the technology. Such binders may be calcareous binders of carbonate hardening, in particular, dolomite lime. Due to their properties, calcareous binders when interacting with carbon dioxide are not exposed to extractive substances of the wood, the alkaline environment conversely helps to neutralize such substances and the formed calcium carbonate crystals are deposited on the surface of wood particles contributing to their bonding [10]. The absence of the need for preliminary preparation of wood fibers reduces the production time, and the low water content of the raw material mixture prevents the occurrence of significant deformations during the molding process.

In connection with the foregoing, studies aimed at studying the carbonate hardening of dolomite lime and obtaining effective structural heat-insulating and heat-insulating building products from wood-lime compositions are relevant.

Studies of the possibility of sequestering carbon dioxide into various building products based on binders containing the active phases $\mathrm{CaO}$ and $\mathrm{MgO}$ have shown the high efficiency of the chemical process of carbonization of the hydrated phases of calcium and magnesium oxides [11-15]. At the same time, the formed carbonate phases contribute to a significant increase in the physicomechanical characteristics of the experimental samples in 
a short time frame. It was also found that building materials based on dolomite binder have an increased durability in aggressive environments due to the higher resistance of its hydrated and carbonate magnesium phases. Studies of individual experimental structures made of wood-magnesia composition and stored for 9 years showed intense carbonization of caustic magnesia hydration products with the formation of magnesium hydroxycarbonate which increased the material density, strength, water resistance and reduced creep deformation of the material under load [13]. Carbonization process also has a low sensitivity to the presence of various impurities in the original raw materials which greatly simplifies the process of production of various building products using carbonate hardening technology. From the analysis of literary sources devoted to the study of calcareous hardening of lime binders and their varieties it follows that calcareous binders of carbonate hardening have a high potential which is currently not used by the building materials industry.

Thus, the aim of this paper was to substantiate the possibility of producing efficient structural heat-insulating and heat-insulating building products of carbonate hardening based on sawdust and dolomite lime by studying the formation of the physicotechnical properties of wood-lime experimental samples of various compositions hardening in a carbon-dioxide gas-air medium.

\section{Methods}

For the study, dolomite rock was used with a fraction of up to $10 \mathrm{~mm}$ mined in the Kamennye Bornitsy open-pit mine of the Leningrad Region (OJSC KARIERY DOLOMITOV). Sawdust from coniferous woods with an extractive substance content of up to $2.0 \%$ was used as wood filler. Chemical analysis of the initial dolomite raw material was determined using X-ray fluorescence analysis on an Epsilon 3XLE ED spectrometer (PANalytical). Mineralogical composition of the dolomite binder before and after carbonization was determined using the system of high-temperature synchronous TGA/DTA/DSC analysis of the STA 8000 of the Perkin Elmer Company in the temperature range of $30-1000^{\circ} \mathrm{C}$ at a heating rate of $10^{\circ} \mathrm{C} / \mathrm{min}$ in a nitrogen atmosphere. The microstructure of the carbonized dolomite matrix was studied using VEGA3 and MIRA3 (TESCAN) series scanning electron microscope. For the selection of the optimal composition of wood-lime composition that would provide the required regulatory characteristics of carbonized material, the methods of mathematical planning of the experiment were used. The experimental data were processed using the StatSoft STATISTICA software package. Determination of the physicomechanical properties of the experimental samples was carried out according to standard methods.

\section{Results}

Previous studies have established that as a result of the organization of carbonate hardening of the dolomite binder, it is possible to obtain a carbonized matrix with high physicomechanical properties [16]. Thus, after 90 minutes of carbonization, the compressive strength of the material reached $32.0 \mathrm{MPa}$ with an average density of $1665 \mathrm{~kg} / \mathrm{m}^{3}$. According to the thermal analysis data, the $\mathrm{Ca}(\mathrm{OH})_{2}$ phase completely disappears in the carbonized dolomite matrix, and the amount of $\mathrm{CaCO}_{3}$ increases by the corresponding amount. At the same time, $\mathrm{Mg}(\mathrm{OH})_{2}$ only slightly $(0.7 \%)$ is converted to magnesium bicarbonate. The main part of $\mathrm{Mg}(\mathrm{OH})_{2}(22.0 \%)$ does not enter into a carbonation reaction under given conditions. Microstructural analysis showed that the high values of compressive strength obtained in a short time are due to the fact that the remnants 
of undecomposed dolomite and its calcite component are probably active components that influence the structure formation of this system acting as a 'substrate' for oriented crystallization of newly formed calcite on the surface of dolomite and calcite particles with the formation of strong cohesive contacts thus creating a compact intergrown structure of calcite and dolomite crystals reinforced with magnesium hydroxide crystals that did not react to carbonation. Accordingly, such data suggest that the introduction of wood filler in the form of wood sawdust into the dolomite binder will provide light construction materials with the required normative characteristics.

Dolomite binder was obtained by roasting dolomite rock in a laboratory muffle furnace of the brand SNOL 6.7/1300 for 2,700 s. A dolomite fraction of 5-10 mm was used for roasting. The roasted product was crushed before passing through a $1.25 \mathrm{~mm}$ sieve and sealed with water to obtain hydrate phases of dolomite lime. Mixing of the dolomite binder and sawdust was carried out in a rotary mixer with a rotor speed of $25 \mathrm{~s}^{-1}$. Additionally, finely dispersed dolomite filler was introduced into the raw mix with a fraction of up to $2.5 \mathrm{~mm}$ in various quantities. Test cylinder samples with a diameter and height of $150 \mathrm{~mm}$ were made using the pressing method from prepared raw mixtures in a specially designed mold equipped with tubes for supplying and discharging the carbon dioxide gas-air mixture (CGAM). The pressing force of the samples was $2500 \mathrm{~N}$. After squeezing the raw mix without removing the pressing force, the CGAM was supplied to the mold with $\mathrm{CO}_{2}$ concentration of $\sim 35 \%$ for $240-480 \mathrm{~s}$. The cylinder samples removed from the mold were dried and tested. General view of carbonized samples before and after testing is presented in Figure 1.

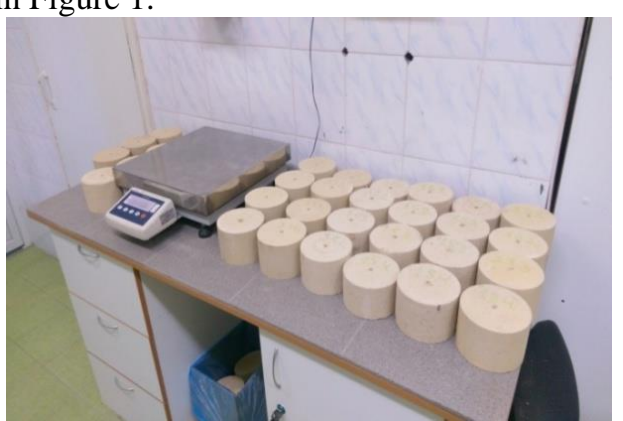

a)

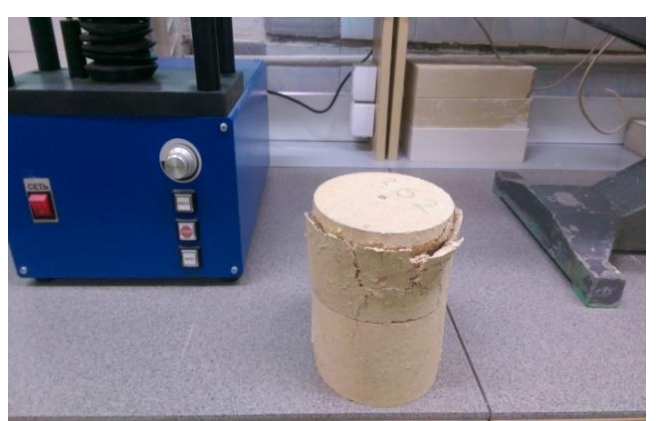

b)

Fig. 1. Determination of the physicomechanical characteristics of the experimental samples: a) determination of average density; b) the sample after determining the compressive strength.

Carbonization as a way to sequester carbon dioxide into various building products has been intensively studied in the last decade [17-20]. It was found that the main factors affecting the speed and completeness of the passage of the reaction are the initial water content of the raw mix, the concentration of carbon dioxide and, to some extent, the conditions for forming the sample. Optimization of the parameters for obtaining wall products based on lime was carried out for composites containing only mineral aggregate. Introduction of organic wood filler requires a review and clarification of the parameters for the manufacture of experimental samples. To establish the necessary parameters for obtaining, the following variable factors were taken as the main ones: water content of the raw mix, \%, amount of sawdust, $\%$. The initial experimental data and the levels of variation of the factors are presented in Table 1. 
Table 1. The levels of variation of the factors of the experiment.

\begin{tabular}{|l|c|c|c|}
\hline \multirow{2}{*}{ Factors } & \multicolumn{2}{|c|}{ Levels of variation } & \multirow{2}{*}{ Interval of variation } \\
\cline { 2 - 3 } & -1 & +1 & 15 \\
\hline Amount of sawdust, \% & 10 & 40 & 2.5 \\
\hline Water content of the mix, \%, by weight & 20 & 25 & \\
\hline
\end{tabular}

These factors were taken as the basis because water content of the raw material mixture primarily affects the process of the interaction of carbon dioxide with calcium hydroxide and magnesium. The adhesion between the wood fibers and in many respects the compressive strength of the samples depends on the completeness of the carbonization reaction. The amount of sawdust determines the density and deformability of the finished products. To determine the optimal ratio of variable factors, 3 series of two-factor experiments were performed. Each series differed in the composition of the mineral part of the raw mix which was varied by the following ratios 'dolomite lime: dolomite filler': $1: 5$, $1: 4$ and $1: 3$. Such proportions were adopted to determine the minimum amount of the binder. The parameters studied were: compressive strength, MPa; average density, $\mathrm{g} / \mathrm{cm}^{3}$; water absorption by weight, $\%$. The results of experimental studies are presented in Fig. 2 and 3 .

I)

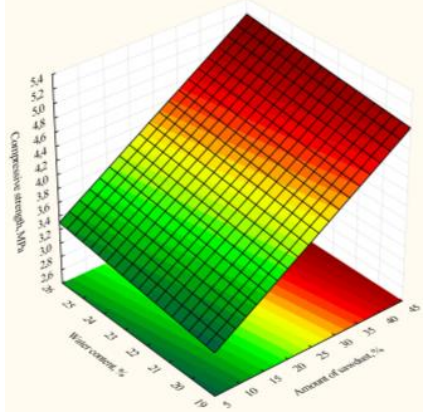

II)

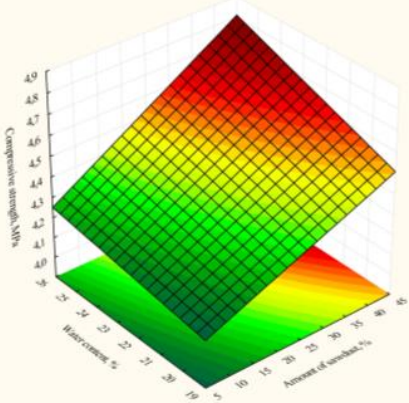

III)

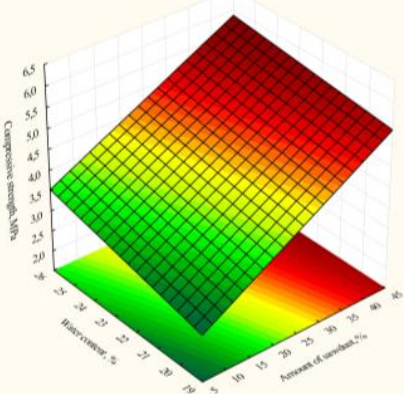

Fig. 2. Change in compressive strength (MPa) of carbonized experimental samples depending on the water content of the raw mix and the amount of sawdust in various proportions 'dolomite lime : dolomite filler' in the mineral part: I) $1: 5$; II) $1: 4$; III) $1: 3$.

I)

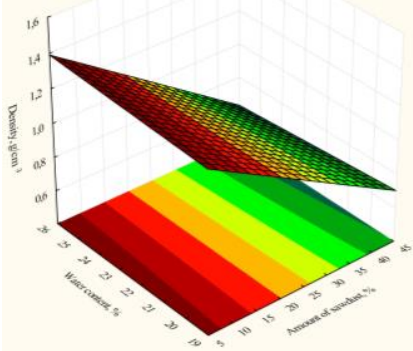

II)

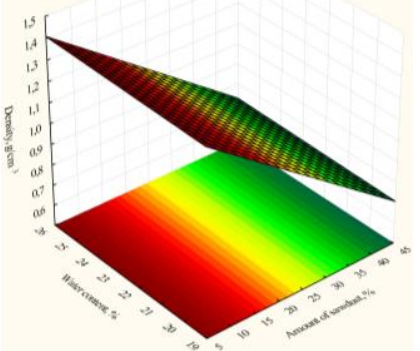

III)

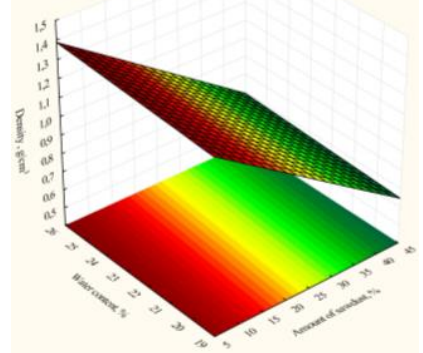

Fig. 3. Change in density $\left(\mathrm{g} / \mathrm{cm}^{3}\right)$ of carbonized experimental samples depending on the water content of the raw mix and the amount of sawdust in various proportions 'dolomite lime: dolomite filler' in the mineral part: I) $1: 5$; II) $1: 4$; III) $1: 3$.

The analysis of the experimental data showed that the amount of the binder in the mineral part of the raw mixture does not significantly affect the index of compressive strength of the experimental samples. Thus, with an increase in the content of the binder 
component, the compressive strength of the experimental samples increases on average by $1 \mathrm{MPa}$, i.e. by $15 \%$ under other equal conditions.

The amount of sawdust in the raw mix has a greater impact on the strength of the experimental samples. When the content of sawdust in the mixture is up to $30 \%$, brittle destruction of carbonized samples occurs. With an increase in the amount of sawdust more than $30 \%$ of this destruction is not observed, and therefore the breaking force was fixed at $10 \%$ deformation of the experimental carbonized samples. Thus, with an increase in the amount of sawdust in varying intervals, an increase in compressive strength is observed with a $10 \%$ deformation of the samples.

The water content of the raw material mixtures is a significant factor affecting the compressive strength of carbonized wood-lime samples. Its significance increases with an increase in the number of binders in the experimental samples (see Fig. 2).

Experimental samples with an initial water content of the raw mix $-25 \%$ and sawdust $40 \%$ are characterized by the highest indicators of compressive strength (5-6.5 MPa). At the same time, the density of such samples from the entire series is minimal $-0.73-0.81 \mathrm{~g} / \mathrm{cm}^{3}$. The coefficient of thermal conductivity for these samples was $0.13 \mathrm{~W} / \mathrm{m} \cdot \mathrm{K}$. Water absorption of the experimental samples increases with the amount of sawdust. Within the limits of varying the amount of sawdust, water absorption by weight of the experimental samples is, respectively, from 27 to $65 \%$.

\section{Discussion}

The results obtained are consistent with the general conclusions of previously published studies of the carbonate hardening of various binders containing the active phases of calcium and magnesium oxides. The main technological parameters affecting the rate and degree of carbonization of various phases are determined. Research in this area is consistent with general global trends aimed at developing and introducing various methods for sequestrating carbon dioxide in order to reduce its impact on climate. In particular, the obtained positive research results provide prerequisites for the development and implementation of building materials in the industry for the production of efficient carbonate hardening building products with low $\mathrm{CO}_{2}$ emissions. Widespread introduction of such 'green technologies' will significantly reduce carbon dioxide emissions into the atmosphere and thereby reduce the greenhouse effect on the Earth, as well as fulfill quantitative commitments to limit or reduce $\mathrm{CO}_{2}$ emissions for countries that have signed and ratified the Kyoto Protocol to the UN Framework Convention on Climate Change.

\section{Conclusions}

Experimental studies have confirmed the possibility of obtaining effective structural heatinsulating and heat-insulating construction products based on sawdust and lime binder hardening in carbon dioxide. The process of obtaining samples in a specially designed mold takes 4-8 minutes; after that the samples achieve the required characteristics. The samples extracted from the mold do not require additional exposure and have specified geometrical and physico-mechanical parameters. Compressive strength of experimental samples was 2.0-6.5 MPa depending on the composition of the raw mix. Thus, even with the minimum content of the binder, the compressive strength of the experimental carbonized samples meets the requirements of the standards for lightweight wall materials (heat-insulating and structural heat-insulating). The density of the samples decreases as the amount of sawdust in the raw mix increases and is $0.73-0.81 \mathrm{~g} / \mathrm{cm}^{3}$ with $40 \%$ content of the wood component. 
The coefficient of thermal conductivity for samples containing $40 \%$ of sawdust was $0.13 \mathrm{~W} / \mathrm{m} \cdot \mathrm{K}$ which fully satisfies the requirements for heat-insulating materials.

The study was carried out with the financial support of the university grant V.I. Vernadsky Crimean Federal University scientific project framework No. UG 01/2018.

\section{References}

1. H. Ma, W. Zhou, X. Lu, Z. Ding, Y. Cao Energy Procedia 88, 807-813 (2016)

2. E. Latif, R.M.H. Lawrence, A.D. Shea, P. Walker Energy\&Buildings 165, 76-91 (2018)

3. F. Aldawi, F. Alam, A. Date, M. Alghamdi, F. Aldhawi 67, 403-418 (2013)

4. G.A. Ormondroyd Wood Comp. 47-66 (2015)

5. J.A. Youngquist Wood-base Composites and Panel Products (USDA Forest Service, Madison. WI, USA. 1999)

6. A. Pizzi J. of Adh. Sci. Techn. 20, 829-846 (2006)

7. C. Lacoste, R.El Hage, A. Bergeret, S. Corn, P. Lacroix Carbohydrate polym. 184, 1-8 (2018)

8. S.H. Ghaffar, M. Fan J. of Adh. and Adhes. 48, 92-101 (2014)

9. F.C. Jorge, C. Pereira, J.M. Ferreira Holz roh werkst 62, 370-377 (2004)

10. M.H. Simatupang, C. Habighorst, H. Lange, A. Neubauer Cement \& Concr. Comp. 17, 187-197 (1995)

11. C. Unluer Carbon Dioxide Sequestration in Cementitious Construction Materials, 129173 (2018)

12. P. He, C. Shi, C.S. Poon Carbon Dioxide Sequestration in Cementitious Construction Materials, 411-430 (2018)

13. T.A. Plekhanova, J. Kerieneb, A. Gailiusb, G.I. Yakovlev Constr. and Build. Mater.21, 1833-1838 (2007)

14. H. Zhanga, C. Shena, P. Xi, K. Chen, F. Zhang Constr. and Build. Mater. 185, 609-616 (2018)

15. S. Ruan,C. Unluer Cem. and Concr. Comp. 80, 104-114 (2017)

16. N. Lyubomirskiy, A. Bakhtin, T. Bakhtina IOP Conf. Series: Mater. Sci. and Engin. 365, 032032 (2018)

17. N. Lyubomirskiy, A. Bakhtin, T. Bakhtina IOP Conf. Series: Mater. Sci. and Engin. 463, 2. 032065 (2018)

18. N.V. Lyubomirskiy, S. Fic, S.I. Fedorkin Intern. Conf. on Constr. and Arch.: Theory and Practice of Industry Development (CATPID 2018) 931, 475-480 (2018)

19. N. Lyubomirskiy, S.I. Fedorkin, A.S. Bakhtin, T.A. Bakhtina Malaysian Constr. Res. J. 23 (3), 15-26 (2017)

20. F. Pacheco-Torgal Carbon Dioxide Sequestration in Cementitious Construction Materials, 3-12 (2018) 